\title{
Development of Melanoma and Cancer without Decreasing of Cambial Cells Number in Morphofunctional Zones
}

\author{
Tatiana M. Yavisheva, Sergey D. Shcherbakov \\ Closed Joint Stock Company "R-Pharm”, Moscow, Russia \\ Email: javisheva@rambler.ru
}

Received 1 February 2014; revised 6 April 2014; accepted 19 April 2014

Copyright (C) 2014 by authors and Scientific Research Publishing Inc.

This work is licensed under the Creative Commons Attribution International License (CC BY). http://creativecommons.org/licenses/by/4.0/

(c) (i) Open Access

\begin{abstract}
In age groups from 20 to 60 years old, proliferation and differentiation of cells happen in a morphofunctional zone, in the electric field excited by 12 pairs of mother and daughter cells, turned out at cambial cells division. Thus in daughter cells the Src SH2 domain necessary both for cytoskeleton formation and tyrosinase activization is activated. If the conditions for strengthening of tyrosinase activity are created in organism, the portion of Src participating in the cytoskeleton building can decrease to critical level that will lead to development of a malignant tumor. If action of a stimulating factor is quite strong, proliferation of malignant cells begins at a stage of melanocyte, and a melanoma occurs. If action of factors is long and not strong, more remote descendants of daughter cells proliferate, and a cancer appears. In order that there will be a normal differentiation of malignantly changed daughter cells, it is necessary to block the tyrosinase. Thus all SH2 domains will go on cell cytoskeleton formation.
\end{abstract}

\section{Keywords}

Morphofunctional Zone at People of 20 - 60 Years Old, Tirosinase, Melanoma, Cancer

\section{Introduction}

\subsection{Short Description of Morphofunctional Zone Work}

In our previous works the concept of cell proliferation and differentiation in the system of morphofunctional zones was offered [1] [2]. According to this concept proliferation and differentiation of cells happen in the morphofunctional zone consisting of two subunits, working in turn (Figure 1(a), Figure 1(b)). 


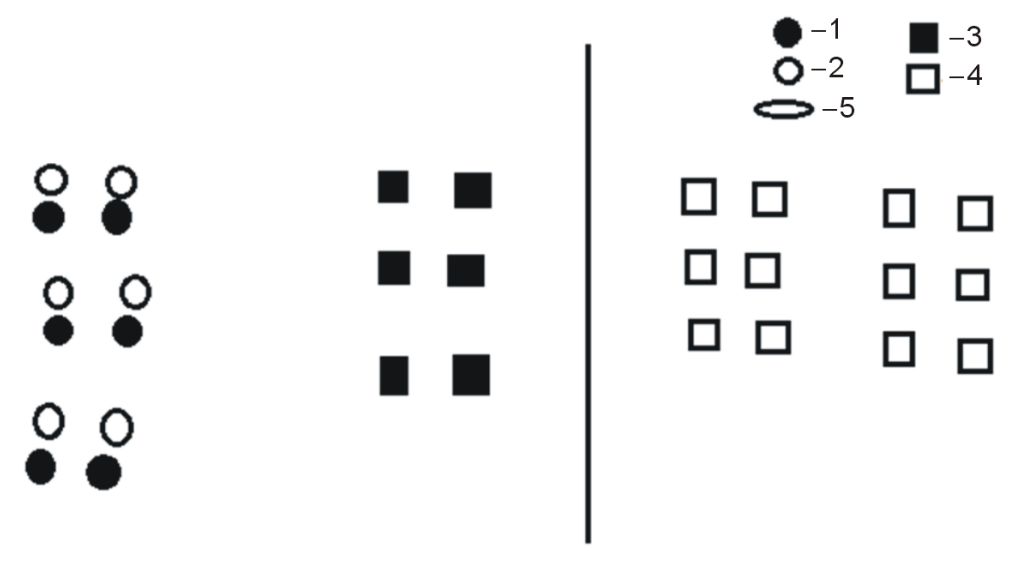

Subunit 1

Subunit 2

(a)

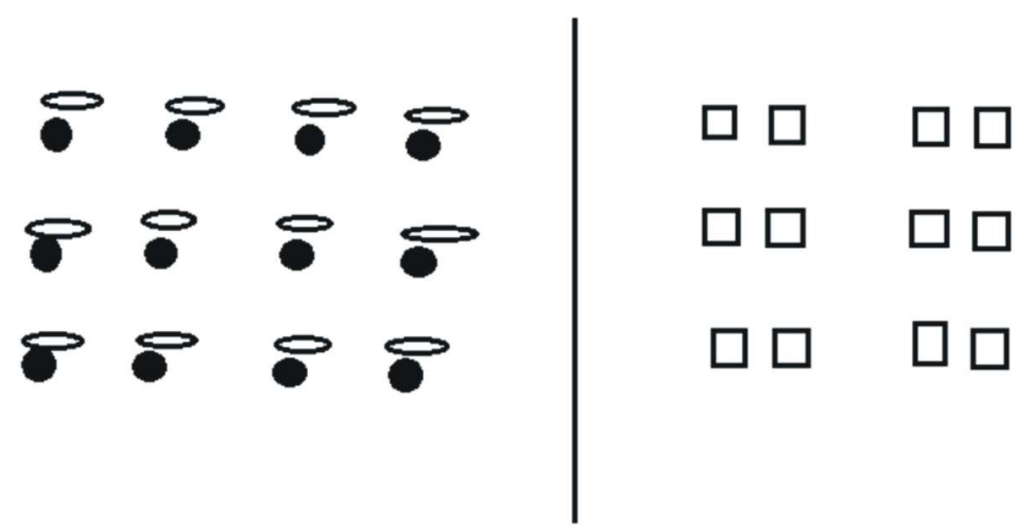

Subunit 1

Subunit 2

(b)

\begin{abstract}
Figure 1. Epithelial morphofunctional zone. (a) Proliferation of the first 6 cambial cells in one zone subunit. Differentiation of daughter cells does not present; (b) Proliferation of all 12 cambial cells of the same zone subunit. The differentiation of daughter cells occurs (scheme). 1-mother cells, 2-daughter cells, 3-activated cambial cells, 4-non-activated cambial cells, 5-differentiated daughter cells.
\end{abstract}

Each subunit at persons of 20 - 40, 41 - 60 years old contains 11 - 12 cambial cells, and over 75 years old -7 cells. At division of cambial cells, mother and daughter cells pairs are formed. The mother cell with the division axis, vertical to the basal membrane is in close proximity to this membrane in which growth factors with spastic action (like a transforming growth factor $\beta$ ) collect. Therefore the genome, having a negative charge, constricts stronger in the mother cell, than in the daughter. It leads to redistribution of superficial charges between two of these cells and to emergence of some electric field. Division of cambial cells in one zone subunit does not happen simultaneously. At first the first 6 cells, and then the other 6 in the same zone subunit divide. The formed daughter cells at division of the first 6 cambial cells are not elongated in electric field, i.e. aren't exposed to a differentiation (Figure 1(a)). Only at division of all 12 cambial cells of the same subunit, there is a differentiation of daughter cells in the electric field, excited by 12 mother and daughter cells pairs (Figure 1(b)). But epithelial cells can't be stretched by the electric field forces without their preliminary cortex relaxation. This function carries out stroma (Figure 2). We showed earlier [2] that stroma has the same morphofunctional zones in which cambial cells divide simultaneously with cambial cells of the epithelium. Function of stroma consists in a relaxation of epithelial cells cortex, constricted due to their own growth factors; therefore epithelial daughter cells have an opportunity to stretch in electric field and to be differentiated. 


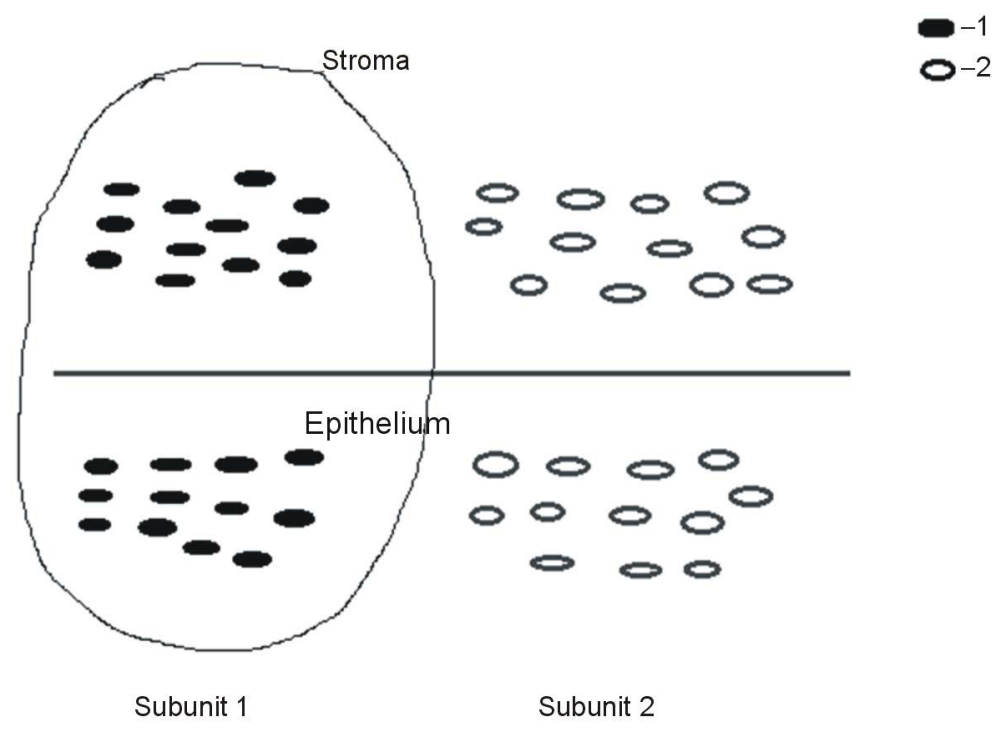

Figure 2. Epithelial-stromal morphofunctional zone in normal epithelial tissue: interaction of stromal and epithelial components of one zone subunit (scheme). 1-activated pairs of mother and daughter cells, 2-non-activated pairs of mother and daughter cells.

Because the electric field formed by 6 mother and daughter cells pairs is not enough for a differentiation of epithelial daughter cells, decrease in quantity of cambial cells in one subunit of the morphofunctional zone to 6 leads to development of a malignant tumor.

\subsection{The Purpose of Our Investigation}

It is shown above that with aging, especially after 75 years old there is a sharp decrease in cambial cells number to 7 that is close to threshold level (6 cells) at which the differentiation of cells is absent. Action of various factors can cause falling of these cells number to critical level that leads to the development of a malignant tumor in such patients. However the tumor may arise at more young patients, when the adverse factors decrease the number of cambial cells in the epithelial morphofunctional zone to 6 . But at these patients the malignant tumor along with it can develop without decrease of cambial cells quantity. Thus there are not clear questions concerning the pathogenesis of a malignant tumor in such cases. Besides, there is a question why these persons in one case have a melanoma, and in others-a cancer. For this purpose we summarized our own experimental material on studying of the normal and pathologically changed epithelial tissue [1] [2], which allowed proving theoretically emergence of such a malignant nosology.

Further, functioning of cambial cells at patients of 20 - 60 years old with normal quantity of cambial cells in each epithelial zone subunit will be considered.

\section{Participation of Src-Kinase and RhoA in Cell Differentiation}

Two key proteins take part in realization of process of cells differentiation in the morphofunctional zone- tyrosine kinase Src and RhoA (one of the small G-proteins) [1]. Stromal daughter cells by means of their growth factors influence over the epithelial cells and make active the Src kinase SH3 domain in them through a number of intermediaries. Then Src phosphorylates p 190 RhoGAP protein which inactivates RhoA. As a result there is a weakening of actin-myosin interaction, that leads to a relaxation of epithelial cells cortex and possibility of their stretching in electric field. But the cortex relaxation is only preparatory stage to the differentiation. The system of microtubules and the intermediate filaments, stretching a nucleus that will cause untwisting of certain loci of the chromosomes responsible for various differentiation, is necessary. It is shown by us that these microfilaments develop in the cells during the stretching of weakened epithelial cells cortex in the electric field. So, stretching of the cell leads to an active splitting off from the Golgi complex membranes of exocytose bubbles for inserting into the plasmatic membrane and to outflowing of the inactive Src localized on these membranes. The 
microtubules are formed at centrosome near Golgi complex, and their nucleation occurs in large protein complexes, which consist of Src, PI3K (3-phosphoinosite-kinase) and $\gamma$-tubulin. Src poorly interacts with $\gamma$-tubulin as has a low affinity to it. Active PI3K interacts with $\gamma$-tubulin, phosphorylating it and making available for interaction with Src. Thus inactive Src associates with active phosphotyrosine $\gamma$-tubulin site by means of the SH2 domain necessary for stable interaction of tubulin dimmers through SH2 group. As a result the kinase site of the $\mathrm{SH} 2$ domain becomes active. In this regard the system of microtubules and the intermediate filaments which formation depends upon microtubules develops. It is known that at the end of a mitosis the chromosomes have the ordered ana-telophase orientation [3], therefore in daughter cells with a vertical division axis, centromeres are located in the upper cell pole and telomeres in the lower pole and in mother cells telomeres settle down in the upper cell pole and centromeres—in the lower pole (Figure 3(a), Figure 3(b)).

That is why formed microtubules and intermediate filaments shift the lower poles of the daughter cells nucleus and stretch them in parallel with the basal membrane. Consequently, the centrosome and telomeres fixed at the nucleus membrane turn from a vertical to a horizontal direction and gather before the leading edge of the epidermal cell. Therewith, chromosomes having certain nucleus periphery-binding sites with the periphery of a cell, are going to stretch nearby telomeres resulting in epithelial cell formation.

So, during the fibroblasts growth factors influence over the daughter cells in the epithelial layer, there is the activation of Src SH3 domain in these cells, necessary for their cortex relaxation, and at stretching in electric field there is an activization of the Src SH2 domain necessary for their cytoskeleton formation and direct epithelial differentiation. Thus, Src expression amplifies in epithelial cells at their stretching, but RhoA remains moderately above Src.

\section{Src and RhoA Define the Proliferation of Cells}

It is shown by us earlier that the ratio of the Src and RhoA expression defines not only a differentiation, but also cell proliferation therefore a differentiation and proliferation-two interconnected processes.

Cells proliferate only in the case if RhoA expression in them exceeds Src more than moderate (Figure 4) [2]. It is caused by that the Src kinase inactivates RhoA therefore the raised expression of RhoA protein leads to that the most part of RhoA remains active. As a result, more formins and microtubules providing a mitosis are formed. Therefore in the epithelial cells where RhoA is moderate above Src, only light strengthening of RhoA expression is necessary for cell proliferation. As a result of it, an epithelium is a fast proliferating tissue. If RhoA sharply exceeds the Src, reaching the threshold level, cell division is blocked due to the spasm of cells. In various epithelial tissues there is an unequal expression of RhoA in relation to Src, therefore they have different

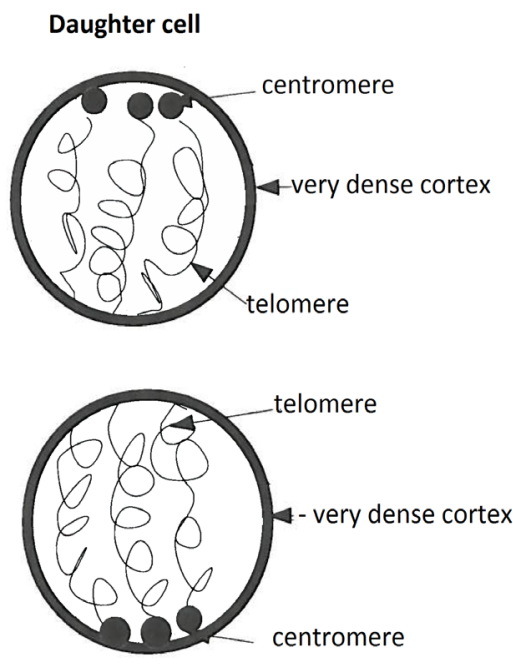

Mother cell
Epidermal daughter cell

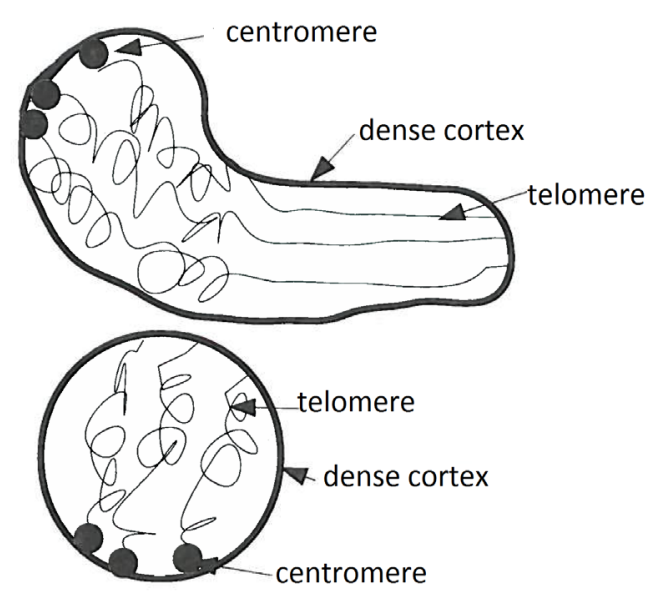

Mother cell

(a)

(b)

Figure 3. The scheme of a cambial cell exit from a mitosis (a) and of an epidermal daughter cell formation (b). 


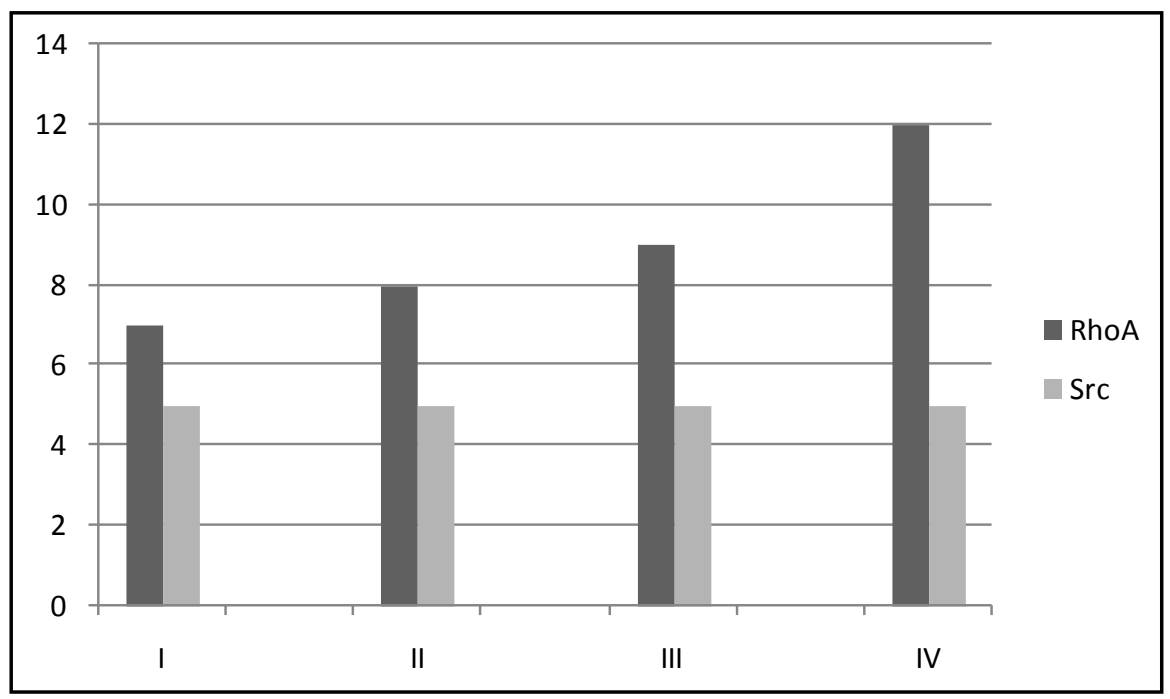

Figure 4. The scheme of RhoA and Src-kinase expression, causing proliferation of cells. I III-proliferation of cells intensifies at gradual increase of RhoA expression in relation to Src; IV—proliferation isn’t present if RhoA expression sharply exceeds Src expression.

proliferative activity. The more RhoA exceeds the Src level, without reaching thus threshold level, the proliferative activity of such tissue is higher.

\section{Participation of Src SH2 Domain in Melanogenesis of a Cell and Definition of Human Melanin Type: Eumelanin or Pheomelanin}

Simultaneously with stretching, black-brown pigment granules, and a dense network of thin processes in cytoplasm of a daughter cell appear, testifying to activization of tyrosinase and melanogenesis in it [4]. If stretching of a daughter cell is absent that is observed at once after cambial cell division, there is no tyrosinase staining. Therefore, melanogenesis begins at the time of cell stretching and the expression of Src SH2 domain in it, i.e. this domain takes direct part in the transcription and tyrosinase activization. Really, in epithelial cells RhoA is moderate above Src that causes stretching of these cells and untwisting of DNA loops closer to telomeres and defines their epithelial differentiation [1]. Therefore in the same sites there is possible the transcription of the genes coding tyrosinase. Tyrosinase activation requires its phosphorylation which happens in the area, connected with Golgi complex [5]. Considering the fact of tyrosinase activation at the time of stretching, it is possible to draw a conclusion that phosphorylation is carried out at the expense of active Src which is located here. Therefore, degree of the Src SH2 domain expression defines a transcription and activization of tyrosinase. Therefore, various constitutional level of Src kinase expression in the organism will define prevalence of eumelanin or pheomelanin at the person.

So, normal level of a constitutional Src expression will provide existence of a large number of active tyrosinase which will catalyze a hydroxylation of tyrosine in DOPA and DOPA oxidation in DOPA-quinone, leuco-DOPA-chrome (Figure 5). Besides, high tyrosinase activity will oxidize sulphhydril groups of proteins, thereby will block proteins, without allowing them to join the forming melanin. Really, protein joining would change the configuration of forming melanin and would weaken its interaction with enzyme. Absence of the connection with proteins gives the chance for further formation of the 5,6-dihidroxyindole which polymerization leads to eumelanin creation.

Pheomelanin is formed at people at whom the constitutional Src kinase expression is at lower level, than at people with the eumelanin. The smaller quantity of active tyrosinase which will be able to oxidize the tyrosine only to the DOPA-quinone stage will be formed thus (Figure 6).

Cysteine joins DOPA-quinone then by means of sulphhydril group because small quantity of tyrosinase can't oxidize and inactivate this group. Cysteine joining will change the DOPA-quinone configuration that will stop the further melanogenesis. As a result, 5-S-cysteinyl-DOPA is formed which is monomeric unit of pheomelanin polymer. It is interesting to note that melanosomes with the eumelanin have more elongated form, than with 

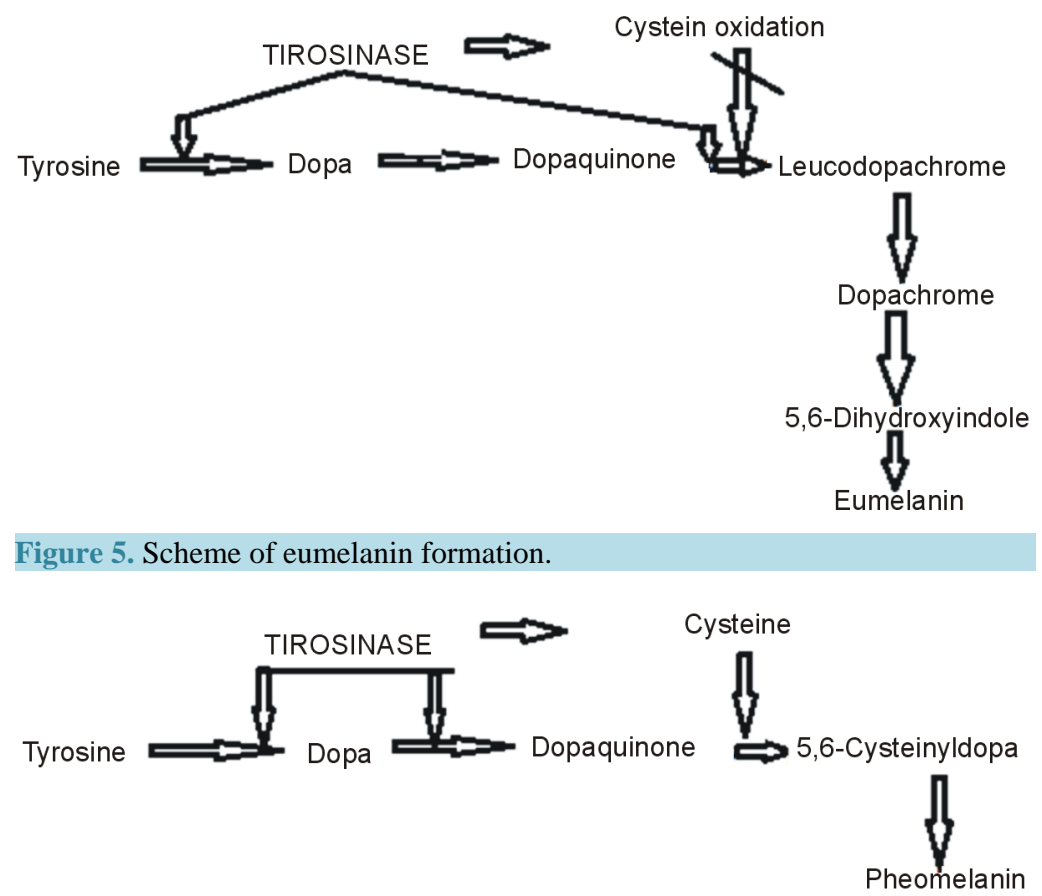

Figure 6. Scheme of pheomelanin formation.

pheomelanin that testifies that genetically programmed Src activity in the people with pheomelanin is less expressed that leads to activization of RhoA, which strengthens the spasm of these structures. Also, the tyrosinase incorporation into melanosome at eumelanin formation happens at later stages of melanosome development, than at pheomelanin. Really, at normal constitutional activity of Src which isn't sharply reducing RhoA there will be a regular splitting of the premelanosomes from smooth membranes of granular endoplasmic reticulum. Therefore formed tyrosinase will incorporate into melanosome of certain maturity. In case of the reduced constitutional Src activity, the RhoA activity will increase, that will lead to strengthening of a spasm of endoplasmic reticulum membranes and to delay in the premelanosome splitting. In this regard, formed tyrosinase will incorporate into melanosome, having earlier stages of the development.

So, in epithelial tissue, cambial cells division passes through a stage of melanocyte (daughter cell) formation. We saw this stage not only in epidermis, but also in the limbic germ zone of eye cornea. However this phase of the cambial cell division in the cornea met extremely seldom as was very short due to the high proliferative activity of corneal epithelium.

Thus the Src kinase SH2 domain which is so necessary for microtubules and intermediate filaments formation, participates not only in assembly of a cytoskeleton and cell differentiation, but also in production and activization of tyrosinase, necessary for melanogenesis in epithelial daughter cells.

In the process of melanocyte (daughter cell) motion in the direction to basal cells, the activity of RhoA due to the microenvironment amplifies in it, and the expression of Src falls. Therefore, melanocytes in basal layer will lose the tyrosinase activity. Therefore action of the various agents strengthening or weakening tyrosinase synthesis depends on what link their action is directed on Src or RhoA.

\section{The Possible Mechanism of Melanoma and Cancer Formation with Normal Quantity of Cambial Cells in Morphofunctional Zone}

It has been mentioned earlier by us that cell proliferation and differentiation happen in a morphofunctional zone on which work various factors have influence: physical, chemical, hormonal, traumas, age [2]. If during the action of these factors the cambial cells quantity in age groups of 20 - 60 years old decreases to threshold level (6 cells), the differentiation of daughter cells is absent and there is a malignant tumor. Thus in daughter cells there is no sufficient activization of Src SH2 domain, necessary for their differentiation. With age the cambial cells number in each zone subunit gradually decreases and reaches 7 cells at people over 75 years old, that is also 
close to threshold level and increases risk of a malignant tumor emergence at elderly people. Therefore, action of the various factors causing carcinogenesis, is directed as a result on decrease in the expression of Src SH2 domain in the daughter cell to the critical level, insufficient for development of cell cytoskeleton and its stretching, and for this purpose there cannot be an obligatory decrease in the number of cambial cells. Really, many tumors arise at people who has no yet age-related decrease in cambial cells number.

So, in age groups of 20 - 40, 41 - 60 years old at normal quantity of cambial cells (12 cells) tyrosinase synthesis can increase under the influence of any factors. Therefore in these conditions Src utilization will be increased for tyrosinase phosphorylation that will reduce participation of Src SH2 domain in cytoskeleton formation. If the portion of this domain decreases to critical level, the differentiation will be absent and there will be a malignant tumor. The effect will be the same, as well as at decrease in cambial cells number. It happens more often at people with constitutional low level of Src kinase activity. At these people the strengthening of tyrosinase activity will lead to decrease the participation of initially not high level of SH2 domain in the cell differentiation. Therefore people at whom prevails pheomelanin, get sick with a melanoma and a cancer of skin more often. If the factor activating tyrosinase is very strong (for example, powerful UF radiation), then at considerable decrease of the SH2 domain, the RhoA expression will sharply increase in the cell cytoskeleton. It is shown above that increase in certain range of RhoA in relation to Src in a cell leads to strengthening of the proliferation, Thus, at powerful UF radiation when the portion of Src SH2 domain considerably decreases, and RhoA raises, daughter cells, having very weak differentiation, will begin to proliferate actively at the earliest stages of the development (melanocyte), that will lead to melanoma development (Figure 7). If action of activating factors not strong and long, activity of the SH2 domain gradually decreases. Therefore, the malignancy and active proliferation will appear in more remote descendants of daughter cells, which have no the tyrosinase activity already, and there will be a cancer. Thus, daughter cells (melanocytes), turned out at once after cambial cells division and having tyrosinase activity, actively proliferate in melanoma. At a cancer more remote descendants of the daughter cells (epithelial cells) which have lost tyrosinase activity, intensively divide. But in both cases a source of malignant regeneration are the daughter cells having tyrosinase activity which represent so-called tumor stem cells.

The period of the greatest estrogen activity (30 - 50 years old) when the influence of estrogen over tissue amplifies is especially dangerous, because estrogens directly activate Src kinase and therefore increase tyrosinase activity of cells.

It is interesting to note that the proliferative activity of tissue is higher, the stage of formation of mother and daughter cells pairs and consequently of melanocyte is shorter. Therefore, in the fast proliferating tissues, such

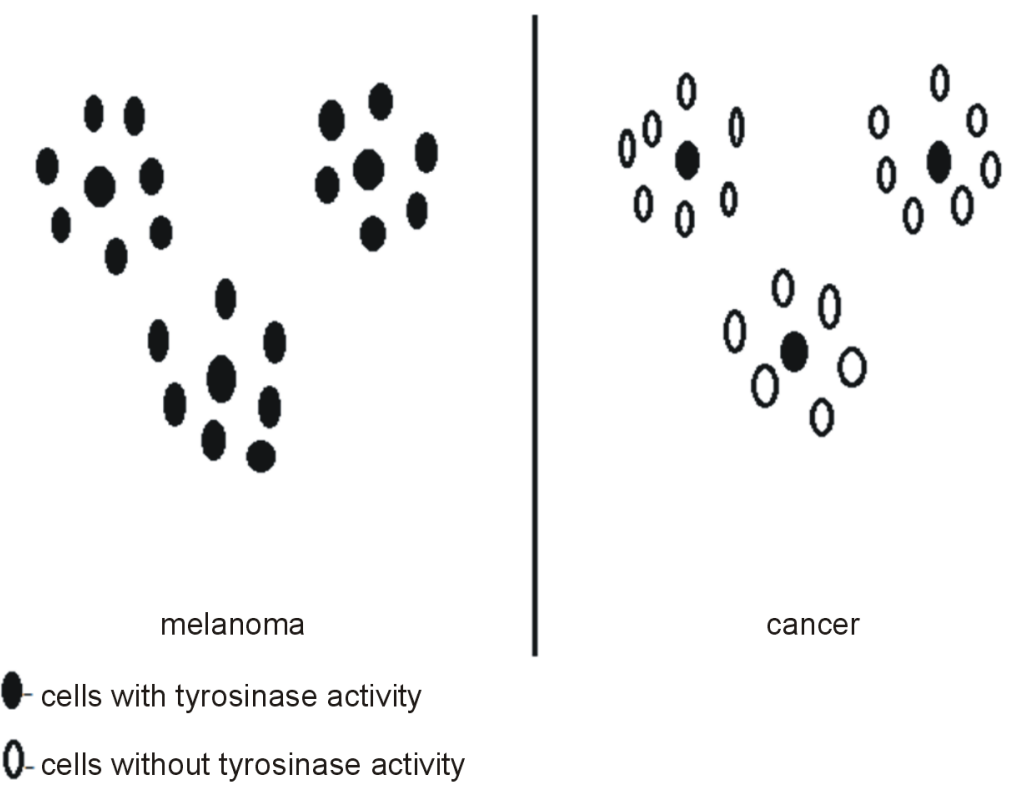

Figure 7. Melanoma and cancer development concerning tyrosinase activity of daughter cells. 
as intestines epithelium, melanoma arises seldom because melanocytes very quickly lose tyrosinase activity and pass into a stage of epithelial cell. In the hair bulb the melanoma does not arise, because division speed of epithelial cells of a germ layer is very great and concedes only to blood cells.

\section{The Possible Way of Strengthening of Src SH2 Domain in the Tumor Cell}

So, in tumor tissue the degree of Src SH2 domain activation isn't sufficient for a differentiation. Therefore, to remedy this situation, it is necessary to strengthen an expression of this domain in these cells. Because this domain participates simultaneously in tyrosinase activazation and in cytoskeleton assembly, it is necessary to block the tyrosinase, so all the quantity of SH2 domains will be used for the cytoskeleton creation for the purpose of a differentiation. One of tyrosinase blockers is glutathione, containing sulphhydril group (SH) at the expense of cysteine, being in its composition. Glutathione is in the restored and oxidized form in the cell. Restoration of oxidized glutathione happens due to the sources of hydrogen developed in the course of a metabolism in the organism. One of glutathione functions is binding of cupriferous enzymes. It is known that the tyrosinase active center contains two cations of copper, each of which is directed by means of three gistidine remnants. The imidazole group of a histidine in a molecule of tyrosinase interacts with copper cations, providing functional activity of enzyme. However SH group are distinguished by exclusively high reactionary ability [6]. Competitively forcing out imidazole group, SH group of glutathione will interact with atom of copper, thus there is formation of mercaptides. But glutathione is quite easily restored in the cell that can lead to its detachment from the tyrosinase active center and activation of the latter. To support interaction of glutathione with tyrosinase and its blockage, it is necessary to block restoration of glutathione by its contact with various acceptors of electrons, for example, iodine, being the most physiologic for the organism. Therefore, iodine introduction into the organism will lead to tyrosinase blockage, and all Src SH2 domains thus will be used only at differentiation, therefore malignantly changed daughter cells which are the centers of tumor growth will be differentiated that will cause the termination of malignant process.

\section{Conclusion}

Thus, the malignant tumor can arise, both at the reduced quantity of cambial cells and at normal, if thus in daughter cells (melanocyte), there is a decrease in the expression of Src SH2 domain, responsible for a differentiation and melanogenesis simultaneously, to critical level. Thus, the melanoma develops as a result of daughter cells proliferation, which is least differentiated, and which has turned out at the first cambial cell division, and a cancer-at proliferation of more remote daughter cells descendants having some signs of a differentiation. Therefore blocking of tyrosinase activity in pathologically changed daughter cells will lead to mobilization in them of endogenous Src and to its participation in the cells differentiation as at a melanoma, and a cancer. The effect of exogenous Src apparently will be incomplete and unstable as in epithelial tissue; the part of it will be used for melanogenesis, reducing the portion of it participating in cytoskeleton formation. Besides, considering a very short period of formation of mother and daughter cells pairs, there is no guarantee that the daughter cells which are the center of tumor growth will be the target of exogenous Src.

\section{References}

[1] Yavisheva, T.M. and Shcherbakov, S.D. (2011) Characteristic Features of Proliferation and Differentiation of Cambial and Daughter Cells in Morphofunctional Zones in Normal Epithelium and Cancer in Age Aspect. Advances in Gerontology, 1, 50-56. http://dx.doi.org/10.1134/S2079057011010140

[2] Yavisheva, T.M. and Shcherbakov, S.D. (2014) Morphofunctional Zones: Structure and Functions. LAMBERT Academic Publishing, Germany.

[3] Shchapova, A.I. (1971) About Structure of a Karyotype and an Order of the Arrangement of Chromosomes in the Interphase Nucleus. Tsytologia, 13, 1157-1163.

[4] Yavisheva, T.M. Shcherbakov, S.D., Golubeva, I.S., Savluchinskaya, L.A. and Rizhova, N.I. (2012) The Relationship between the Epidermal Melanocytes, Langerhans Cells and Epidermal Cambial Cells. International Journal of BioMedicine, 2, 228-231.

[5] White, A., Handler, P., Smith, E., Hill, R. and Lehman, I. (1981) Principles of Biochemistry. Mir, Moscow. (in Russian)

[6] Torchinsky, Yu.M. (1971) Sulphhydrile and Disulphide Groups. Nauka, Moscow. 